\title{
FORMATION OF A DYNAMIC CONSTRUCTION MODEL FOR VIBROACOUSTIC DiAgNOSTICS
}

\author{
Davorka Šaravanja \& Šimun Bogdan
}
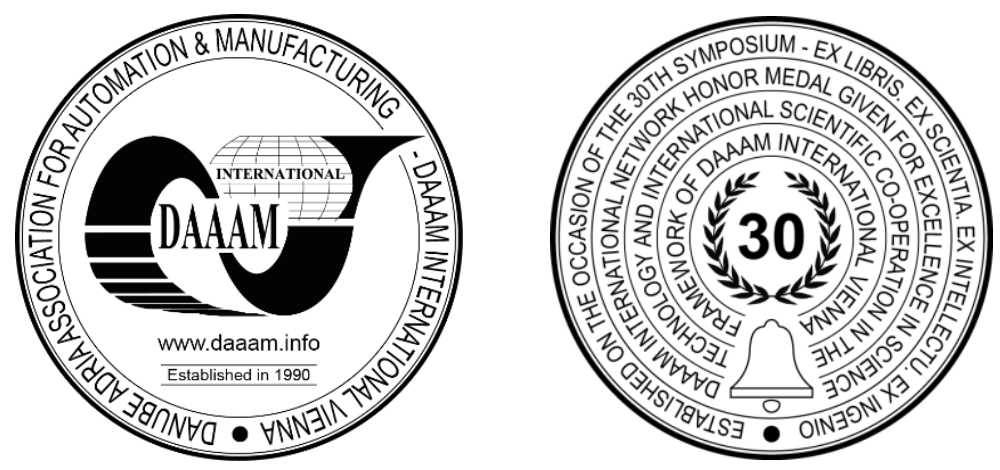

This Publication has to be referred as: Saravanja, D[avorka] \& Bogdan, S[imun] (2020). Formation of a Dynamic Construction Model for Vibroacoustic Diagnostics, Proceedings of the 31st DAAAM International Symposium, pp.0027-0034, B. Katalinic (Ed.), Published by DAAAM International, ISBN 978-3-902734-29-7, ISSN 1726-9679, Vienna, Austria

DOI: $10.2507 / 31$ st.daaam.proceedings.004

\begin{abstract}
In precision rotary machines, rotary elements play the most important role because, during the operation of the machines, they are influenced by loads, forces and moments. This influence causes their gradual damage and the creation of vibration-acoustic waves, which, therefore, can be both primary and secondary sources of problems and defects within rotary machines. It is therefore important to locate, monitor and analyze vibrations in order to diagnose the overall technical condition of the machine. The size and change in the size of the defect can be detected by using the methods of vibroacoustic diagnostics, especially on the elements that are most damaged, and these are usually bearings with cylindrical elements. This paper will show the formation of a dynamic model of a structure under the influence of vibroacoustic waves and the determination of the equations of motion. The thesis is proved by the expression for the calculation of the velocity of propagation of different types of elastic waves and the values of constant spatial, temporal damping coefficients and damping in decibels per unit length for various types of waves in massive structures.
\end{abstract}

Keywords: vibroacoustic diagnostic; dynamic model; elastic waves; equations of motion

\section{Introduction}

To determine the technical condition and precision in the operation of mechanical systems with rotating elements as objects that are diagnosed and presented in the form of models, it is necessary to observe the structural and physical representations of such objects [1]. Such models, which reflect the most basic laws of physical and mechanical processes, which occur in the object in the process of its operation and diagnosis, provide the possibility of choosing the vector component, which describes the construction and choice of functional model operator type [2][3]. It is important to emphasize that experience shows that it is not necessary to observe a construction model of a rotational system in which all phenomena would participate simultaneously. The absence of reciprocity between the state parameters allows to observe the partial construction models of the corresponding interconnections of the object.

The aim of this paper is to show that the knowledge gained after the formation of a dynamic model of machine design, knowledge about the characteristics of the excitation source and the propagation of elastic waves as a result of changes in vibroacoustic parameters, can be used in effective vibroacoustic protection procedures still in the design phase. 


\section{Conditions for vibroacoustic diagnostics of mechanical systems}

Mechanical systems that occur in mechanical engineering (usually as an integral part) have rotary machines of various types and shapes. Therefore, it is very important to pay some attention to these machines in the pre-design phase, because in such mechanical systems hidden potential sources of vibration are hidden, sources that generate various forms of irregularities, the presence of which emphasizes the need to optimize vibration isolation on foundations [4][5][6]. Machines and structures, in general, in terms of the transmission of sound vibrations in them, represent a group of plates and rods interconnected in a certain way. In doing so, vibroacoustic waves, which are generated in machines under the action of dynamic forces created by the interaction of individual parts, propagate from the place of origin in the form of elastic waves [7][8].

Unlike environments such as air and water, where waves of only one type are possible (so-called compression waves), in solids different types of elastic waves can form and propagate. In a body with infinitely many degrees of freedom, there are two types of waves: compression waves with propagation velocities [9][10].:

$$
C_{1}=\sqrt{\frac{E(1-\sigma)}{\rho(1+\sigma)(1-2 \sigma)}}
$$

and shear waves with propagation velocities:

$$
\mathrm{C}_{2}=\sqrt{\frac{\mu}{\rho}}
$$

where $\mathrm{E}$ is the modulus of elasticity; $\sigma$ - Poisson's ratio; $\rho$ - density; $\mu$ - shear modulus [11].

In a solid body of limited dimensions, these two types of waves occur together and form different combinations of mixed longitudinal-shear or longitudinal-transverse waves. Since there are three types of waves in plates: bending, longitudinal and transverse, and in rods there are longitudinal, bending and torsional waves, vibrational energy by the construction of a mechanical system consisting of plates and rods is transmitted in the form of elastic waves: longitudinal, bending, transverse and torsional [12][13].

When longitudinal waves propagate along the plate, its cross section vibrates in the direction of motion of this wave, and the derivatives of the cross sections (directions in that plane) remain their directions [14][15][16]. The equation of motion of the plate, along which longitudinal waves propagate along the $\mathrm{x}$ coordinate, has the form:

$$
\frac{\partial^{2} \xi(\mathrm{x}, \mathrm{t})}{\partial \mathrm{x}^{2}}-\frac{1}{\mathrm{C}_{1}^{2}} \frac{\partial^{2} \xi(\mathrm{x}, \mathrm{t})}{\partial \mathrm{t}^{2}}=\frac{\left(1-\sigma^{2}\right)}{\mathrm{Eh}} \mathrm{P}(\mathrm{x}, \mathrm{t})
$$

where: $\xi$ - displacement of the plate cross section along the $\mathrm{x}$ axis; $\mathrm{C}_{1}$ - velocity of propagation of longitudinal waves; $\mathrm{P}$ $(\mathrm{x}, \mathrm{t})$ - external forces acting on the plate in its plane; $\mathrm{h}$ - plate thickness.

The solution of the differential equation (3) in the form of free (natural) vibrations, in case the external forces are equal to zero [17], can be given in general form by the equation:

$$
\xi(\mathrm{x}, \mathrm{t})=\int_{-\infty}^{\infty} \int_{-\infty}^{\infty} \mathrm{A}\left(\mathrm{k}_{l}, \omega\right) \mathrm{e}^{\mathrm{ik} l_{l} \mathrm{x}-\mathrm{i} \omega \mathrm{t}} \mathrm{dk} \mathrm{k}_{l} \mathrm{~d} \omega
$$

where: $\mathrm{k}_{1}$ - wave number; $\omega$ - circular frequency.

Equation (3) has several solutions, ie the solution of the homogeneous part of the differential equation (3) (one of the mathematical physics equations) has several forms. In the case of transverse vibration, the solution is often assumed in the form of a product of two functions, one of which depends on $\mathrm{x}$, i.e. X (x) and the other which depends on time $t$, i.e. $\mathrm{T}(\mathrm{t})$. It can then be written:

$$
\xi(x, t)=X(x) T(t)
$$

and the differential equation becomes:

$$
\mathrm{X} \ddot{\mathrm{T}}-\mathrm{C}_{1}^{2} \mathrm{X}^{\prime \prime} \mathrm{T}=0
$$


and it can be written in the form:

$$
\frac{\mathrm{X}^{\prime \prime}}{\mathrm{X}}=\frac{1}{\mathrm{C}_{1}^{2}} \frac{\ddot{\mathrm{T}}}{\mathrm{T}}=\lambda, \quad \lambda=\text { const. }
$$

That $\lambda$ is a constant follows from the realization that the left side does not depend on time $\mathrm{t}$ and the right on the $\mathrm{x}$ coordinate, so equality of relations is possible if each of them is a constant. Then it is possible to write two equations:

$$
\mathrm{X}^{\prime \prime}=\lambda \mathrm{X}, \quad \ddot{\mathrm{T}}=\mathrm{C}_{1}^{2} \lambda \mathrm{T}
$$

When the transformation of the differential equation is performed and the above expressions are included, a differential equation is obtained with the solution:

$$
\begin{aligned}
& \frac{\mathrm{d}^{2} \mathrm{~V}\left(\mathrm{ik}_{l}, \mathrm{t}\right)}{\mathrm{dt}^{2}}=\left(\mathrm{ik}_{l} \mathrm{C}_{l}\right)^{2} \mathrm{~V}\left(\mathrm{ik}_{l}, \mathrm{t}\right) \\
& \mathrm{V}\left(\mathrm{ik}_{l}, \mathrm{t}\right)=\mathrm{A}\left(\mathrm{ik}_{l}\right) \mathrm{e}^{\mathrm{ik}_{l} C_{l} \mathrm{t}}+\mathrm{B}\left(\mathrm{ik}_{l}\right) \mathrm{e}^{-\mathrm{ik}_{l} \mathrm{C}_{l} \mathrm{t}}
\end{aligned}
$$

where constants $\mathrm{A}\left(\mathrm{ik}_{l}\right) \mathrm{i} \mathrm{B}\left(\mathrm{ik}_{l}\right)$ are obtained from the initial conditions:

$\xi(\mathrm{x}, 0)=\mathrm{f}(\mathrm{x}) \mathrm{i} \frac{\partial \xi(\mathrm{x}, 0)}{\partial \mathrm{t}}=0$ for values $-\infty \leq \mathrm{x} \leq \infty$.

The transformation of the initial conditions gives:

$\mathrm{F}_{\mathrm{x}}[\xi(\mathrm{x}, 0)]=\mathrm{V}\left(\mathrm{ik}_{l}, 0\right), \mathrm{F}_{\mathrm{x}}[\mathrm{f}(\mathrm{x})]=\mathrm{F}\left(\mathrm{ik}_{l}\right), \mathrm{F}_{\mathrm{x}}\left[\frac{\partial \xi(\mathrm{x}, 0)}{\partial \mathrm{t}}\right]=\frac{\partial}{\partial \mathrm{t}} \mathrm{F}_{\mathrm{x}}[\xi(\mathrm{x}, 0)]=\frac{\partial \mathrm{V}\left(\mathrm{ik}_{l}, 0\right)}{\partial \mathrm{t}}$

so it is $\mathrm{V}\left(\mathrm{ik}_{l}, 0\right)=\mathrm{F}\left(\mathrm{ik}_{l}\right), \frac{\partial \mathrm{V}\left(\mathrm{ik}_{l}, 0\right)}{\partial \mathrm{t}}=0$, and the constants will have values $\mathrm{A}\left(\mathrm{ik}_{l}\right)=\mathrm{A}\left(\mathrm{ik}_{l}\right)=\frac{\mathrm{F}\left(\mathrm{ik}_{l}\right)}{2}$, and it is $\mathrm{V}\left(\mathrm{ik}_{l}, \mathrm{t}\right)=\left(\mathrm{e}^{\mathrm{ik}_{l} \mathrm{C}_{l} \mathrm{t}}+\mathrm{e}^{-\mathrm{ik} \mathrm{C}_{l} \mathrm{t}}\right) \frac{\mathrm{F}\left(\mathrm{ik}_{l}\right)}{2}$.

The inverse transformation according to the table from Fourier transforms will be:

$$
\xi(\mathrm{x}, \mathrm{t})=\frac{1}{2 \pi} \int_{-\infty}^{\infty} \mathrm{V}\left(\mathrm{ik}_{l}, \mathrm{t}\right) \mathrm{e}^{\mathrm{i} k_{l} \mathrm{x}} \mathrm{dk}_{l}=\frac{1}{4 \pi} \int_{-\infty}^{\infty}\left(\mathrm{e}^{\mathrm{i} \mathrm{k}_{l} \mathrm{C}_{l} \mathrm{t}}+\mathrm{e}^{-\mathrm{i} \mathrm{k}_{l} \mathrm{C}_{l} \mathrm{t}}\right) \mathrm{F}\left(\mathrm{ik}_{l}\right) \mathrm{e}^{\mathrm{i} \mathrm{k}_{l}^{\mathrm{x}}} \mathrm{dk}_{l}
$$

This equation gives the solution of the problem for each $\mathrm{f}(\mathrm{x})$, and by applying the translation property we directly obtain:

$$
\xi(x, t)=\frac{1}{2}\left[f\left(x+C_{l} t\right)+f\left(x-C_{l} t\right)\right]
$$

The obtained equation represents the dependence on the motion of the wave. When the shear wave propagates along the plate along the $\mathrm{x}$ coordinate, the cross sections of the plate vibrate in their planes in a direction perpendicular to the direction of wave motion. In doing so, the cross-sectional derivatives also remain straight lines [12].

The equation of motion of the plate, which performs shear vibration, in this case will be [18]:

$$
\frac{\partial^{2} v(\mathrm{x}, \mathrm{t})}{\partial \mathrm{x}^{2}}-\frac{1}{\mathrm{C}_{\mathrm{p}}^{2}} \frac{\partial^{2} v(\mathrm{x}, \mathrm{t})}{\partial \mathrm{t}^{2}}=\frac{1}{\mathrm{Gh}} \mathrm{P}(\mathrm{x}, \mathrm{t})
$$

where $v$ is the displacement of the cross section of the plate, parallel to its plane, in the direction perpendicular to the $\mathrm{x}$ axis; $C_{p}$ - shear wave propagation velocity in the plate; $G$ - plate shear modulus; $P(x, t)$ - external forces acting on the plate in its plane. Longitudinal and shear deformations in the plate can be interchanged, i.e. the existence of resistance in the plate leads to the conversion of the shear wave into longitudinal and vice versa. 
By spreading on the plate (rod), the bending waves cause, in addition to the transverse displacement $\xi(t)$ of each cross section, its rotation $\theta$ (t) [19]. This is the following connection between these parameters:

$$
\theta(\mathrm{x})=\frac{\partial \xi(\mathrm{x})}{\partial \mathrm{x}}=\xi^{\prime}(\mathrm{x})
$$

The bending vibration is the result of the appearance of internal forces, namely: the transverse force $\mathrm{Q}$ ( $\mathrm{x}$ ), which is perpendicular to the plate surface, and the bending moment $\mathrm{M}(\mathrm{x})$ [20], which acts in a plane perpendicular to the plate surface and oriented along the $\mathrm{x}$ coordinate with displacement $\xi(\mathrm{x})$ expressions:

$$
\mathrm{Q}(\mathrm{x})=\frac{\mathrm{D} \partial^{3} \xi(\mathrm{x})}{\partial \mathrm{x}^{3}}=\mathrm{D} \xi^{\prime \prime \prime}(\mathrm{x}) ; \mathrm{M}(\mathrm{x})=-\frac{\mathrm{D} \partial^{2} \xi(\mathrm{x})}{\partial \mathrm{x}^{2}}=-\mathrm{D} \xi^{\prime \prime}(\mathrm{x})
$$

where $\mathrm{D}$ is the flexural stiffness of the plate. Flexural rigidity of the plate, units $[\mathrm{Nm}]$, is given by the expression $\mathrm{D}=\frac{\mathrm{Eh}^{3}}{12\left(1-\mu^{2}\right)}=\frac{\mathrm{E}^{*} \mathrm{~h}^{3}}{12}, \quad \mathrm{E}^{*}=\frac{\mathrm{E}}{\left(1-\mu^{2}\right)}$ and is analogous to the bending stiffness of the beam $\mathrm{B}=\mathrm{EI}_{\mathrm{x}}$ at unit section width. $E^{*}$ modified modulus of elasticity, $\mu$-Poisson's ratio. When the bending wave propagates across the plate, the distribution of the deformation phases is transmitted along the same plate with the phase velocity of the bending wave $\mathrm{C}_{\mathrm{s}}$. The value of this speed is given by the expression:

$$
\mathrm{C}_{\mathrm{S}}=\sqrt{\left(\omega \mathrm{C}_{1} \mathrm{r}\right)}=\sqrt[4]{\left(\frac{\mathrm{D} \omega^{2}}{\mathrm{~m}}\right)}=\sqrt[4]{\left(\frac{E I \omega^{2}}{\mathrm{~m}\left(1-\sigma^{2}\right)}\right)}
$$

where: $r$ - radius of inertia of cross-section $r=\sqrt{\frac{I}{S}}=\sqrt{\frac{I}{h}}=\frac{h}{12}, m$ - mass per unit area; E - modulus of elasticity; $\sigma$ Poisson's ratio; $\mathrm{C}_{1}$ - velocity of longitudinal wave propagation.

The cross-sectional area $S$, as well as the value of the moment of inertia I are calculated per unit cross-sectional width, i.e. $S=$ h. As can be seen from formula (18), the phase velocity of a bending wave depends on the frequency $\omega$, i.e. there is a dispersion of its values [19]. It can be seen from the above expressions that all quantities, which determine the bending wave in a plate, are ultimately determined by the transverse displacement of its cross section $\xi(\mathrm{x})$.

The equation of motion in the one-dimensional case has the form:

$$
\mathrm{D} \frac{\partial^{4} \xi(\mathrm{x}, \mathrm{t})}{\partial \mathrm{x}^{4}}+\mathrm{m} \frac{\partial^{2} \xi(\mathrm{x}, \mathrm{t})}{\partial \mathrm{t}^{2}}=\mathrm{F}(\mathrm{x}, \mathrm{t})
$$

where $\mathrm{F}(\mathrm{x}, \mathrm{t})$ is the function of the distribution of the external load on the surface of the plate and its change with time. In the case of carrying out the process of vibration isolation, i.e. the process of damping the vibration energies in the plate [21], the equation of motion becomes:

$$
\mathrm{D} \frac{\partial^{4} \xi(\mathrm{x}, \mathrm{t})}{\partial \mathrm{x}^{4}}+\mathrm{m} \frac{\partial^{2} \xi(\mathrm{x}, \mathrm{t})}{\partial \mathrm{t}^{2}}+\mathrm{R} \frac{\partial \xi(\mathrm{x}, \mathrm{t})}{\partial \mathrm{t}}=\mathrm{F}(\mathrm{x}, \mathrm{t})
$$

After differentiation by time of the homogeneous equation of harmonic bending vibration, the expression is obtained:

$$
\xi^{\mathrm{IV}}(\mathrm{x})-\frac{\omega^{2} \mathrm{~m}}{\mathrm{D}}(1-\mathrm{i} \eta) \xi(\mathrm{x})=0
$$

where is $\eta=\frac{R}{\omega m}$. 
As with real constructions $\eta^{2}=1$, expression (20) can be transformed into the form:

$$
\xi^{\mathrm{IV}}(\mathrm{x})-\frac{\omega^{2} \mathrm{~m}}{\mathrm{D}(1+\mathrm{i} \eta)} \xi(\mathrm{x})=0
$$

The magnitude $\eta$ is a characteristic of the energy absorption of the bending waves in the plate. Formally, energy losses can be taken into account by a complex representation of the modulus of elasticity: $\bar{E}=\mathrm{E}(1+i \eta)$, where $\eta \mathrm{E}$ is the modulus of loss.

In the experiments where the above calculation is applied, a model was used whose components are an additional plate and a shaft with elements (simulation of the most common form of rotary system from practice), and the use of bending vibration equations is applied to solve Krilov functions [22]. On the example of a homogeneous equation of bending vibrations without losses, the basic regularities of propagation of bending waves in a plate (rod) were considered.

By applying Krilov's functions or, the so-called function of letters - S, T, U and V, the solution of the equation can be obtained in the form:

$$
\xi(x)=A_{1} e^{-j k_{s} x}+A_{2} e^{j k_{s} x}+A_{3} e^{-k_{s} x}+A_{4} e^{k_{s} x}
$$

The first two terms of expression (22) describe moving waves, which propagate in opposite directions. The last two members correspond to inhomogeneous bending waves, which do not participate in the energy transfer since they are rapidly damped. The amplitude of inhomogeneous bending waves decreases e times at the distance $\lambda_{\mathrm{s}} / 2 \pi$ from the place of origin. Inhomogeneous waves occur near external loads or obstacles in the path of their propagation.

Different interconnections of plates and rods represent artificial barriers to bending waves in machine constructions. The transformation of bending waves into waves of other types (longitudinal, shear, torsional) must be taken into account, not only when the plane bends perpendicularly to an obstacle, but also when approaching at an angle [19] [16].

The property known as vibration field diffusion in machine constructions simplifies the solution of many problems related to the propagation of sound waves. For a field to be considered diffuse, it must satisfy the conditions of isotropy and homogeneity (a field with the same properties in all directions and at all points). These conditions can be met in limited constructions due to multiple bounce of the bending waves from the ends.

The vibrational behavior of finite-length rods is very similar to the vibrational behavior of finite-length plates [23]. However, in infinite or semi-infinite rods, bending waves can exist at all frequencies, in the case of rods of finite lengths 1 , free waves can exist only at frequencies where the boundary conditions are satisfied, i.e. at the natural frequencies of free bending vibration. Thus, for rods with articulated ends (no displacement and no bending moments) $\xi=0 \mathrm{i} \xi^{\prime \prime}=0$, the natural frequencies are given by the expression:

$$
\omega_{\mathrm{n}}=\frac{\pi^{2} \mathrm{n}^{2}}{1^{2}} \sqrt{\frac{\mathrm{EI}}{\mathrm{m}}}, \quad \mathrm{n}=1,2,3, \ldots
$$

The totality of its own frequencies forms a discrete spectrum.

For a rod with free ends $\omega_{n}=\frac{\pi^{2} n^{2}}{1^{2}} \sqrt{\frac{E I}{m}}, \quad n=1,2,3, \ldots$, ie. on those where there is no bending moment and transverse force, the natural frequencies are found using the formula (it is interesting to note that the frequency equation is the same for a beam clamped on both sides):

$$
\omega_{\mathrm{n}} \approx \frac{\pi^{2}(2 \mathrm{n}+1)^{2}}{41^{2}} \sqrt{\frac{\mathrm{EI}}{\mathrm{m}}}, \quad \mathrm{n}=1,2,3, \ldots
$$

A comparison of expressions (23) and (24) shows that, at $2 \mathrm{n} \square 1$ the values of the natural frequency density at the ends of the articulated support rod and the free-end rod, they practically coincide, i.e. it can be argued that at a sufficiently large number $n$, the natural frequency density of the bending vibration rod ceases to depend on boundary conditions. Each of its own frequencies corresponds to the form of vibration of the rod. For the articulated support rod, this form is presented: 


$$
\xi_{\mathrm{n}}(\mathrm{x})=\mathrm{A}_{\mathrm{n}} \operatorname{sink}_{\mathrm{n}} \mathrm{x}
$$

where $A_{n}$ is the amplitude of the free vibrations of the plate at the frequency $\omega_{n}$. The totality of natural frequencies and their corresponding forms of free vibrations of the rod is commonly called modes. The function, which describes the form of the mode is called fundamental and is denoted by $\psi_{\mathrm{n}}(\mathrm{x})$. In a given case it will be:

$$
\psi_{\mathrm{n}}(\mathrm{x})=\operatorname{sink}_{\mathrm{n}} \mathrm{x} .
$$

Tab.1 gives expressions for calculating the propagation velocity of different types of elastic waves, and Tab.2 gives the values of constant spatial $\gamma$ and time $\delta$ damping coefficients [24] , as well as the damping $D_{1}$ in decibels per unit length for different types of waves.

\begin{tabular}{|l|l|l|l|}
\hline Wave type & $\begin{array}{l}\text { Speed of energy } \\
\text { propagation }\end{array}$ & Wave type & $\begin{array}{l}\text { Speed of energy } \\
\text { propagation }\end{array}$ \\
\hline bending waves in the plate & $\mathrm{C}_{\mathrm{s}}=2 \sqrt{\omega \mathrm{C}_{\mathrm{l}} \mathrm{h} / \sqrt{12}}$ & bending waves in a rod & $\mathrm{C}_{\mathrm{s}}=2 \sqrt{\omega \mathrm{C}_{1} / \sqrt{\mathrm{I} / \mathrm{S}}}$ \\
\hline longitudinal waves in the plate & $\mathrm{C}_{1}=\sqrt{\mathrm{E} /\left[\rho\left(1-\sigma^{2}\right)\right]}$ & $\begin{array}{l}\text { longitudinal waves in } \\
\text { the rod }\end{array}$ & $\mathrm{C}_{1}=\sqrt{\mathrm{E} / \rho}$ \\
\hline Shear waves in a plate & $\mathrm{C}_{\mathrm{p}}=\sqrt{\mathrm{G} / \rho}$ & torsional waves in a rod & $\mathrm{C}_{\mathrm{t}}=\sqrt{(\mathrm{G} / \rho) \gamma}$ \\
\hline
\end{tabular}

Table 1. Calculation formulas for estimating the propagation velocity of elastic waves in massive machine structures

The vibration field can be considered diffuse if five modes are excited simultaneously. Since with increasing frequency the frequency spectrum of the plate mode (rod) becomes relatively higher density (Fig.1), this vibration field can be considered diffuse above some frequency f. For massive constructions of machine aggregates, the vibration field can be considered diffuse in the frequency range above $1 \mathrm{kHz}$ [25].

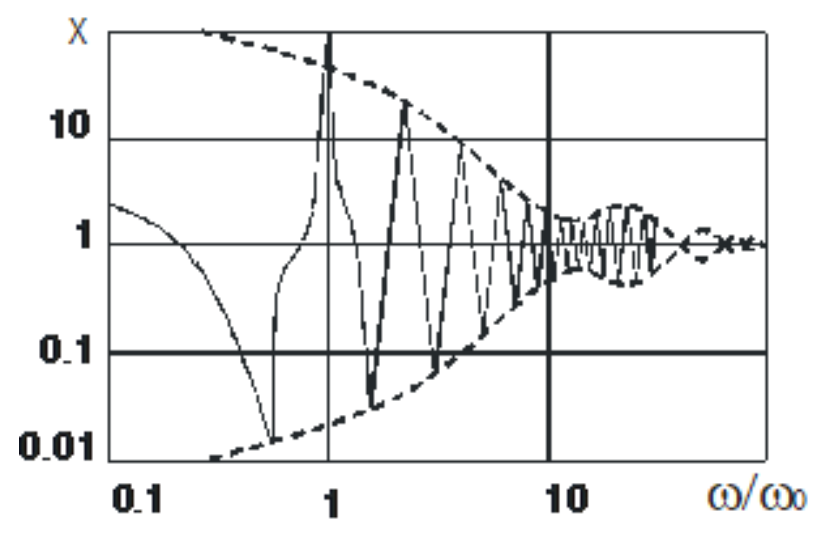

Fig. 1. Spectrum of plate/rod natural frequencies

\begin{tabular}{|l|l|l|l|}
\hline Wave type & $\begin{array}{l}\text { Spatial damping } \\
\text { coefficient } \gamma\end{array}$ & $\begin{array}{l}\text { Time damping } \\
\text { coefficient } \delta\end{array}$ & $\begin{array}{l}\text { Decreasing the } \\
\text { amplitude per unit } \\
\text { length } D_{1}\end{array}$ \\
\hline Bending waves in a plate or rod & $\pi \eta / 2 \lambda_{\mathrm{s}}$ & $\pi / \eta$ & $13.65 \eta / \lambda_{\mathrm{s}}$ \\
\hline $\begin{array}{l}\text { Longitudinal waves of a plate or } \\
\text { rod }\end{array}$ & $\pi \eta / \lambda_{1}$ & $\pi / \eta$ & $27.3 \eta / \lambda_{1}$ \\
\hline Shear waves in a plate & $\pi \eta / \lambda_{p}$ & $\pi / \eta$ & $27.3 \eta / \lambda_{\mathrm{p}}$ \\
\hline Torsional waves in a rod & $\pi \eta / \lambda_{\mathrm{t}}$ & $\pi / \eta$ & $27.3 \eta / \lambda_{\mathrm{t}}$ \\
\hline
\end{tabular}

Table 2. Coefficients of spatial and temporal damping of elastic waves in massive structures

In the zone of low frequencies in the distribution of vibrational energy by structures, the decisive role belongs to the first modes. 


\section{Conclusion}

Massive constructions of machines, which have vibration isolating properties, set certain requirements in relation to the placement of sensors in the construction when solving diagnostic problems. Experience shows that the loss of vibration energy during propagation through the structure leads to a decrease in the level of the useful signal, which carries information about the technical condition of the assembly, therefore the sensor must be placed as close to the source of vibration in the diagnosed assembly as possible.

When investigating the regularity of excitation and propagation of vibroacoustic vibrations in complex machine aggregates, it is important to take into account the final goal of the research. For proper vibroacoustic diagnostics it is necessary to form a dynamic model of construction and when solving a diagnostic problem, it is important to choose not only information points for setting the encoder, but also to assess the character of vibration excitation in the event of defects, set the diagnostic mode and determine the frequency range in order to obtain characteristic diagnostic indicators.

It should be emphasized that when solving the problem of vibroacoustic diagnostics in complex machine aggregates, one should not be guided by assumptions about the possibility of establishing an adequate dynamic model of the diagnosed object in a wide frequency range and hope for the possibility of extracting valuable diagnostic information about the technical condition of the mechanical object. The mathematical apparatus of these equations is specially developed for the object control system with processes, which are slow enough and is absolutely unsuitable for quantitative evaluation of vibroacoustic characteristics of machines in the high frequency range.

This approach of forming a dynamic model for determining the propagation velocities of elastic waves between structural elements as part of the overall method of vibroacoustic machine diagnostics helps to eliminate unexpected failures, fractures, ensure normal operation and reduce costs of each kind. In this case, the source of relevant information about the technical condition of rotary machine assemblies are the propagation velocities of elastic waves, whose changes are in certain correlations with changes in technical condition parameters caused by the gradual weakening of machine construction elements.

\section{References}

[1] https://lpsa.swarthmore.edu/Systems/MechRotating/RotMechSysAll.html

[2] Barton, P.I. (1997). Introduction Industrial Experience With Dynamic Simulation, Department of Chemical Engineering Massachusetts Institute of Technology Cambridge, MA, Paul I. Barton, July 1997, Available from: http://web.mit.edu/yoric-locker/BartonDynamicSimNotes.pdf

[3] Šaravanja, D.; Petković, D. (2020). Technical Diagnostics -Methods of Testing the Structure and Condition of the System, University of Mostar, ISBN 978-9958-16-131-5, COBISS.BH-ID 29005574, Mostar

[4] Fundamentals of mechanical engineering, IDC Technologies Pty LtdPO Box 1093, IDC Technologies 2009. ISBN: 978-1-921007-07-1, Available from: https://www.eit.edu.au/cms/resources/books/fundamentals-ofmechanical-engineering

[5] Šaravanja, D.; Petković, D. (2010). Vibration Diagnostics - Theory and Practice, FSR University of Mostar-MF University of Zenica, ISBN 978-9958-9263-1-0, Mostar-Zenica

[6] Rašković, P.D. (1957). Oscillation Theory, Naučna knjiga, ID: 960613008, Beograd

[7] Fahy, F.; Gardonio, P. (2007). Sound and Structural Vibration, 2007, DOI 10.1016/B978-0-12-373633-8.X5000-5, Available from: https://www.researchgate.net/publication/316087545_Sound_and_Structural_Vibration

[8] Husin, S.; Mba, D. \& R.I. Raja Hamzah, R.I. (2010).Viability of the Application of Acoustic Emission (AE) Technology for the Process and Management of Maintenance in Industries: Defect Detection, On-Line Condition Monitoring, Diagnostic and Prognostic Tools, Available from:https://www.researchgate.net/publication/44260857 Accessed 2020-08-27

[9] Broch, J.T.Mechanical Vibration and Shock Measurements (1984), Brüel \& Kjær, ISBN 878735534 5, Naerum, Denmark

[10] Schubert, A.; Zeidler, H.; Jahn, S.F.; Flemmig, S.; Schulze, R. (2013). Vibration Analysis of an UltrasonicAssisted Joining System, 24th DAAAM International Symposium on Intelligent Manufacturing and Automation, Available from: https://www.daaam.info/Downloads/Pdfs/proceedings/proceedings_2013/134.pdf

[11] https://www.sciencedirect.com/topics/engineering/shear-wave-velocity

[12] https://www.nde-ed.org/EducationResources/CommunityCollege/Ultrasonics/Physics/modepropagation.htm

[13] Korablev, S.S. and others (1989). Vibration Diagnostics in Precision Instruments, Hemisphere Publishing Corporation, ISBN 0891168125, 9780891168126, University of California

[13] Wang, X.; Hopkins, C. (2016). Bending, longitudinal and torsional wave transmission on Euler-Bernoulli and Timoshenko beams with high propagation losses, The Journal of the Acoustical Society of America 140, 2312; doi: 10.1121/1.4963900, Available from: https://asa.scitation.org/doi/pdf/10.1121/1.4963900

[15] https://www.brown.edu/Departments/Engineering/Courses/En4/Notes/Vibrations/Vibrations.pdf

[16] C. W. de Silva (2007). Vibration Fundamentals and Practice, Taylor \& Francis Group, ISBN 0-8493-1987-0, London-New York 
[17] C. W. de Silva (2007). Vibration Fundamentals and Practice, Taylor \& Francis Group, ISBN 0-8493-1987-0, London-New York

[18] Senjanović, I.; Vladimir, N.; Cho, D.S.; Choi , T. M. (2014). Vibration Analysis Of Thick Plates - Analytical And Numerical Approaches, Available from:

https://www.researchgate.net/publication/282819774_Vibration_Analysis_of_Thick_Plates_Analytical_and_Numerical _Approaches Accessed Aug 302020.

[19] Cuenca, J. (2009). Wave Models For The Flexural Vibrations Of Thin Plates, Doctoral thesis in Acoustics Universit'e du Maine, Le Mans, France, Available from: https://tel.archives-ouvertes.fr/tel-00442260/document

[20] Kumar, A. (2018). Free Transverse Vibration Analysis of thin rectangular plates having arbitrarily varying nonhomogeneity along two concurrent edge, Department of Mechanical Engineering National Institute of Technology Rourkela Odisha 769008, Available from: https://arxiv.org/ftp/arxiv/papers/1810/1810.06402.pdf

[21] A.J. Pretlove, H.G. Natke, (1995). Basic vibration theory and its application to beams and plates, Available from:file:///C:/Users/pc/Downloads/1995_Bookmatter_VibrationProblemsInStructures.pdf

[22] file:///C:/Users/pc/Downloads/Beamsonelasticfoundation.pdf Accessed Sep 152020

[23] Ying, Z.G.; N, Y.Q. (2017). Dynamic characteristics of infinite-length and finite-length rods with high-wavenumber periodic parameters, Available from https://doi.org/10.1177/1077546316687676

[24] Nad, M. (2007). Structural dynamic modification of vibrating systems, Available from: https://www.researchgate.net/publication/285022677 Accessed 2020-08-27

[25] Long, M. (2014). Sound and Solid Surfaces, Available from: https://doi.org/10.1016/B978-0-12-398258-2.000076, Accessed 2020-08-28 